\title{
Manejo de la vía aérea mediante intubación submental en fracturas maxilofaciales
}

\author{
DOI 10.5377/alerta.v4i3.11276 \\ Jorge Eugenio Flores Valdez ${ }^{*}$, Ligia Carolina Martínez² , Juan Carlos Arias ${ }^{3}$, Susana Peña Martínez ${ }^{4}$ \\ 1, 2, 3 y 4. Hospital Nacional Rosales, San Salvador, San Salvador. \\ ${ }^{*}$ Correspondencia \\ $\square$ jor flores5@hotmail.com \\ 1. (1) 0000-0003-0911-8956
}

\section{Resumen}

La intubación submentoniana es útil en procedimientos quirúrgicos en donde la intubación nasotraqueal está contraindicada y la intubación orotraqueal no es ideal debido a la fijación intermaxilar. Este informe describe dos pacientes masculinos que se presentaron al Hospital Nacional Rosales, en el servicio de Cirugía Maxilofacial de El Salvador, con historia de sufrir accidente de tránsito. El primer caso evidenció al examen radiológico fractura simple y desplazada de sínfisis mandibular, fractura simple huesos propios nasales y desviación del tabique nasal. El segundo caso es un paciente con fractura de sínfisis y doble cóndilo mandibular que presentaba imposibilidad a la apertura bucal. A través de la técnica del cirujano maxilofacial Hernández Altemir (España), el tubo endotraqueal atraviesa una incisión extraoral en la región submentoniana del suelo de la boca, para poder practicar reducción abierta de fracturas faciales, logrando adecuada oclusión, con mínimos cuidados posoperatorios y con una cicatriz estética. Al terminar la cirugía, la posición del tubo endotraqueal regresa a su posición original y el paciente evoluciona satisfactoriamente.

\section{Palabras clave}

Intubación, manejo de la vía aérea, cirugía maxilofacial, traumatología.

\begin{abstract}
Submental intubation is useful in surgical procedures where nasotracheal intubation is contraindicated and orotracheal intubation is not ideal, due to intermaxillary fixation. This report describes two male patients who presented to the Rosales National Hospital, in the Maxillofacial Surgery service of El Salvador with a history of suffering a traffic accident. The first case, on radiological examination, revealed a simple and displaced fracture of the mandibular symphysis, a simple fracture of the nasal bones, and deviation of the nasal septum. The second case is a patient with a symphysis fracture and a double mandibular condyle that presented an inability to open the mouth. Through the technique of the maxillofacial surgeon Hernández Altemir (Spain), the endotracheal tube passes through an extraoral incision in the submental region of the floor of the mouth, in order to perform open reduction of facial fractures, achieving adequate occlusion, with minimal postoperative care, and with an aesthetic scar. At the end of the surgery, the position of the endotracheal tube returns to its original position, and the patient evolves successfully.
\end{abstract}

Keywords

Intubation, airway management, maxillofacial surgery, traumatology

\section{Introducción}

Los traumas del macizo facial son complejos y representan un desafío para el cirujano, así como para anestesistas y anestesiólogos, debido al área común de trabajo para estas disciplinas médicas, pues es importante mantener continuamente segura la vía aérea del paciente?.
G

Management of the airway through submental intubation in maxillofacial fractures

Citación recomendada: Flores J, Martínez L, Arias J, Peña S. Manejo de la vía aérea mediante intubación submental en fracturas maxilofaciales. Alerta. 2021;4(3):103-108 DOl 10.5377/alerta.v4i3.11276

Recibido:

14 de abril de 202

Aceptado:

5 de julio de 2021

Publicado:

26 de julio de 2021

\section{Contribución de autoría:} JEFV': Cirugía, búsqueda bibliográfica, redacción de artículo. LCM²: controles posquirúrgicos hospitalarios (fotos, radiografías, medicación). JCA3: Búsqueda bibliográfica, redacción y revisión de ambos casos clínicos. SPM ${ }^{4}$ : Redacción y revisión final del caso clínico.

\section{Conflicto de intereses:}

Los autores declaran no tener conflicto de interés. 
En el manejo de la vía aérea existen dos tipos de intubación: se puede utilizar la intubación orotraqueal o la intubación nasotraqueal; sin embargo, se vuelven improcedentes ante situaciones que requieren bloqueo intermaxilar en fracturas mandibulares para lograr la oclusión ideal en el paciente en el caso de la intubación orotraqueal, y en las fracturas de huesos nasales, fracturas naso-orbito-etmoidales del tercio medio facial en el caso de la intubación nasotraqueal. Así mismo, las fracturas de la base del cráneo, con o sin rinorrea, deformación de la anatomía nasal o la falta de experiencia en el equipo de anestesia, tampoco hacen posible practicar la intubación nasotraqueal, requeridas para una reducción de fracturas faciales ${ }^{2,3}$.

En el pasado, ante estos casos se realizaba una traqueostomía o cricotiroidotomía como primera opción para mantener la vía aérea permeable, ya que una intubación compleja, por las condiciones locales de un trauma del complejo maxilofacial, genera algunos riesgos, como mayor trauma de la vía aérea, edema laríngeo, sangrado, entre otros, lo que conduce, además, a una ventilación prolongada ${ }^{1,4}$.

Para superar estas dificultades y posibilitar que las cirugías del macizo facial puedan efectuarse con complicaciones mínimas, el cirujano maxilofacial español Hernández Altemir (1986) propuso una nueva técnica de intubación que implicaba pasar el tubo endotraqueal a través de una incisión extraoral en la región submentoniana del suelo de la boca intraoralmente ${ }^{5}$.

La literatura recomendó que esta técnica de intubación es una medida para asegurar la vía respiratoria sin obstáculos y permitir la fijación intermaxilar, como objetivo principal al realizar la reducción de fractura de la mandibula $a^{3,6,7}$.

Por lo tanto, el presente estudio tiene como objetivo demostrar que la intubación submentoniana es una alternativa efectiva en el manejo de la vía aérea en pacientes con traumas maxilofaciales durante la cirugía y la anestesia general?

\section{Presentación de primer caso}

Se presenta un paciente masculino de 21 años de edad, quien consultó en la unidad de emergencia del Hospital Nacional Rosales, con historia de cuatro horas de evolución de haber sufrido accidente de tránsito (atropellado) y sin antecedentes médicos, quirúrgicos o alérgicos. Al examen médi$\mathrm{co}$, el paciente se encontraba consciente, orientado. Se descartó trauma de tórax y abdomen, se dio manejo conservador por trauma craneocefálico leve cerrado y se interconsultó con la especialidad de cirugía maxilofacial por sospecha de fractura de huesos propios nasales y sínfisis mandibular.

Clínicamente, presentó múltiples abrasiones faciales, desviación del tabique nasal, leve edema en región del mentón y crepitación de los huesos propios nasales. Al examen intraoral presentó extrusión de incisivos centrales superiores, fractura coronal de incisivo lateral superior izquierdo, intrusión de incisivo central inferior izquierdo, movilidad del segmento anterosuperior y disrupción de cortical ósea anterior mandibular. (Figura 1).

El examen radiológico evidenció fractura de sínfisis mandibular compuesta desplaza$d a$, fractura simple de huesos propios nasales y desviación del tabique nasal (Figura 2).

Posteriormente, se indicó tratamiento quirúrgico, para reducción abierta de fractura mandibular y reducción cerrada de huesos propios nasales.

El paciente fue manejado con monitoreo estándar ASA de nivel 1, la inducción anestésica de secuencia rápida se realizó con propofol a 200 mg, fentanilo $200 \mathrm{mcg}$, coadyuvante dexametasona 12 mg, lidocaína 60 mg, relajante muscular no despolarizante (cicatracurio $10 \mathrm{mg}$ ) y relajante muscular despolarizante (succinilcolina $100 \mathrm{mg}$ ). Fue catalogado por el equipo de anestesiología como una vía aérea difícil y se logró la instrumentación de la vía de forma orotraqueal al tercer intento con un tubo endotraqueal reforzado con balón 7.5 $\mathrm{mm}$. Posteriormente, previa asepsia y antisepsia, se infiltró lidocaína al $2 \%$ con vasoconstrictor 1:100 000 y se realizó la incisión en la piel posterior a 10 minutos para respetar el periodo de latencia de la lidocaína. Se practicó la incisión submentoniana lateral y no medial, a $2 \mathrm{~cm}$ lateral derecho a la línea media del mentón, paralela al borde basilar de la mandíbula, para evitar traumatizar los conductos de Wharton e interferir con la inserción de los músculos geniogloso y genihioideo, así como para evitar dañar los vasos linguales ${ }^{1,3,6,7}$.

Se introdujo primero el manguito, a través del túnel, y luego el tubo; después se procedió a insuflar el balón y se fijó a la piel con seda negra 2-0 (Figura 3).

Previo a la fijación del tubo en piel, el equipo de anestesia efectuó auscultación para confirmar la precisión del tubo en tráquea ${ }^{7}$. El mantenimiento de la anestesia se llevó a cabo por medio de la administración de Sevoflurano al 2 \%. La duración prome- 


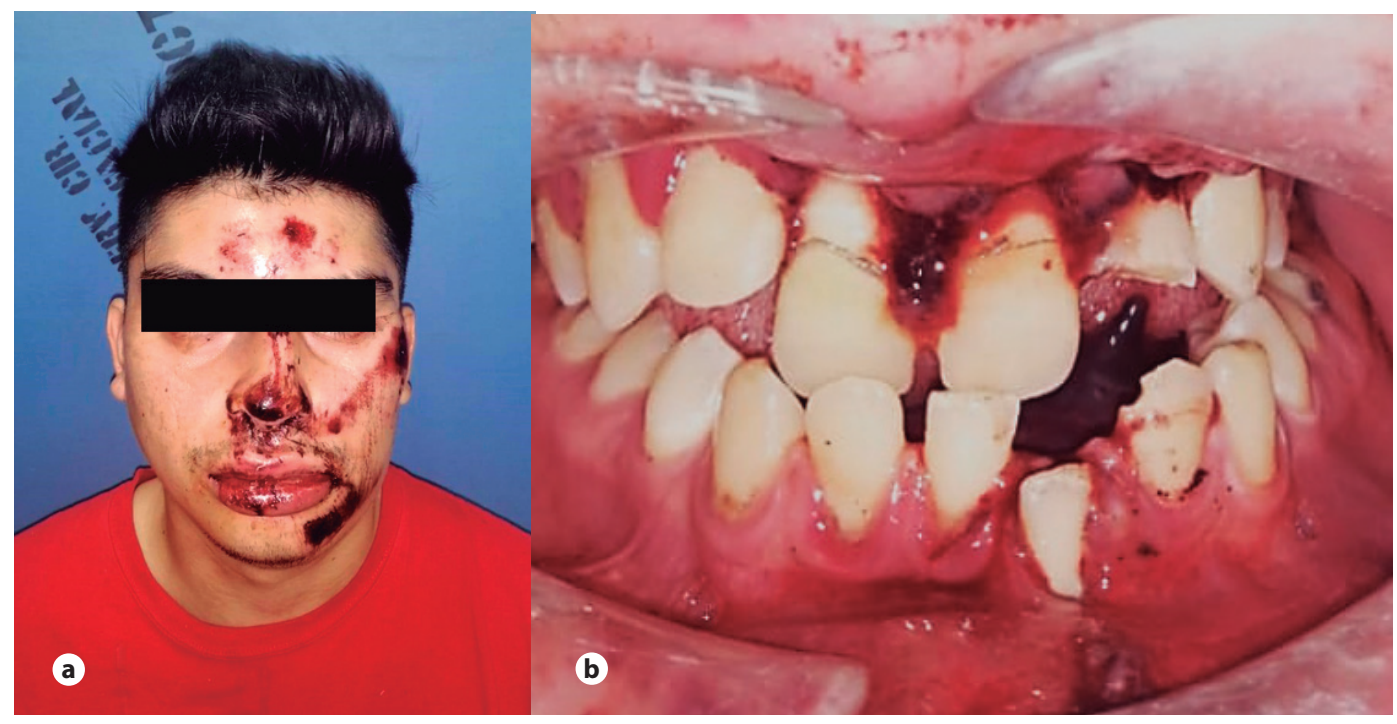

Figura 1a. Paciente con edema generalizado y múltiples abrasiones en región facial. 1b Presenta extrusión de incisivos centrales superiores, fractura coronal de incisivo lateral superior izquierdo, intrusión de incisivo central inferior izquierdo y disrupción de cortical ósea anterior mandibular.

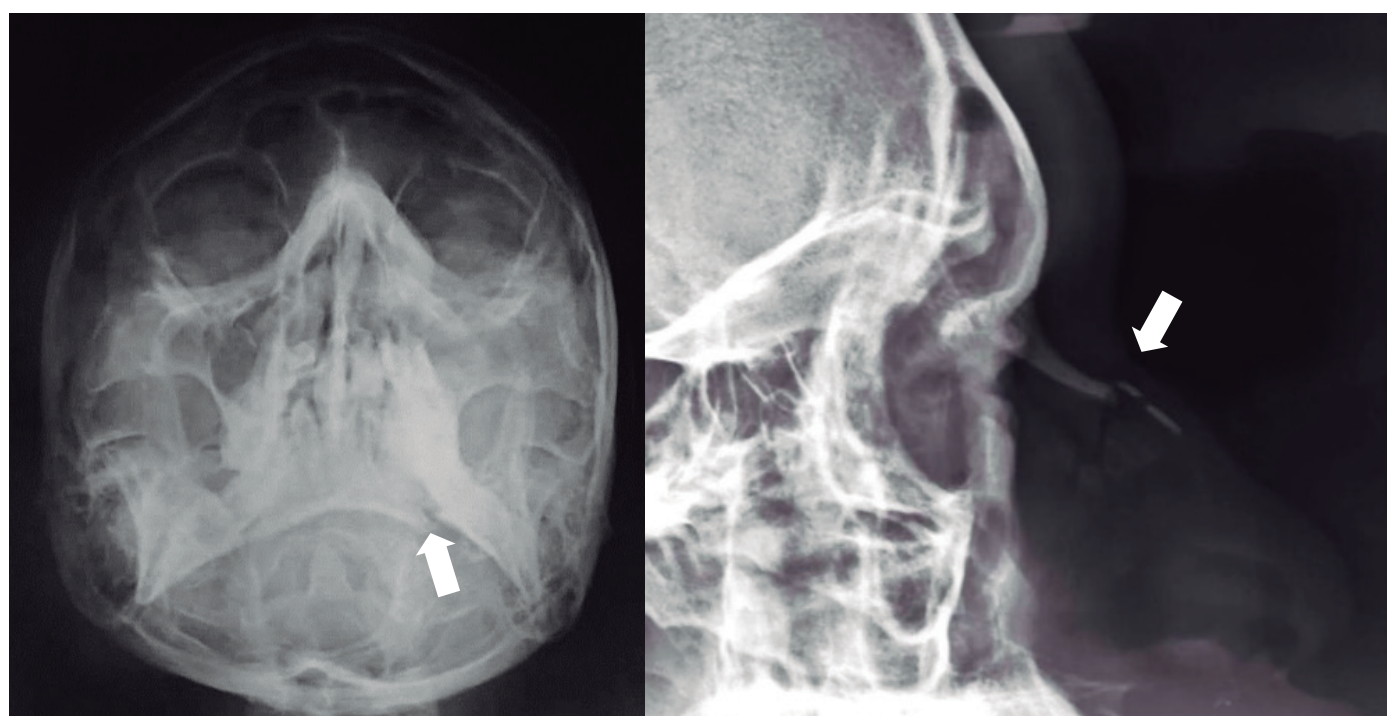

Figura 2a. Radiografía de Waters donde se evidencia una fractura de sínfisis mandibular, 2 b. Radiografía de huesos propios de la nariz donde se evidencia desviación del tabique nasal y huesos propios nasales. Fuente: Servicio de Radiología, Hospital Nacional Rosales.

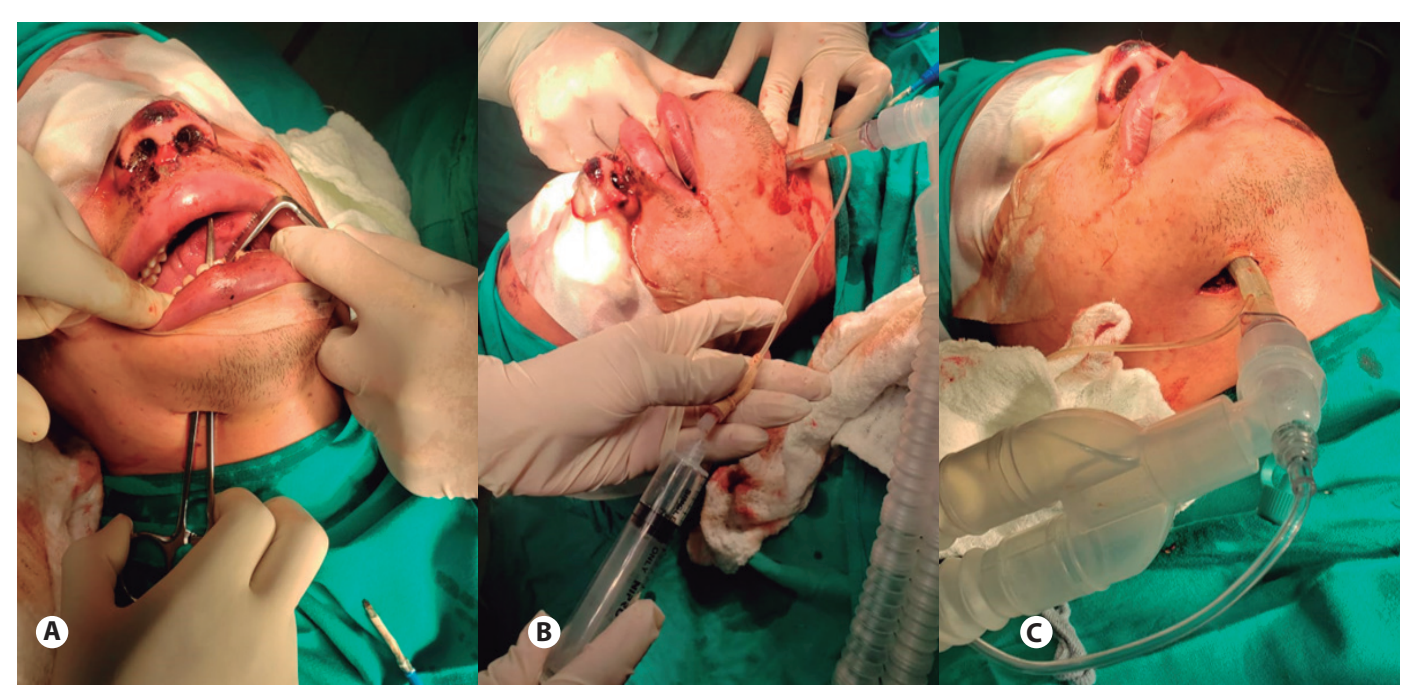

Figura 3a. Se inició con una incisión submentoniana lateral de $2 \mathrm{~cm}$ de longitud, luego se realizó una disección al piso de boca. 3b. Introducción del manguito a través del túnel, del tubo, luego se insufló el balón. 3c. Fijación del tubo con seda negra 2- 0. 
dio del procedimiento fue de aproximadamente siete minutos ${ }^{2,6,7}$. El tiempo medio de interrupción de la ventilación fue de aproximadamente 25 segundos ${ }^{6}$.

Asegurada la vía aérea, se procedió a la reducción abierta de la fractura de la sínfisis mandibular mediante un abordaje vestibular, visualizando el desplazamiento de la fractura; se estabilizó con material de osteosíntesis con miniplacas del sistema 2.0 y se colocaron los tornillos de $5 \mathrm{~mm}$ en la región superior y tornillos bicorticales de $11 \mathrm{~mm}$ en la región basal de la mandíbula. Posteriormente se realizó la reducción cerrada de fractura nasal y la corrección del tabique nasal, finalizando con la colocación del taponamiento nasal anterior, más el uso de una férula nasal por siete días.

El paciente evolucionó con buen alineamiento de la fractura, con apertura oral adecuada y buena estética nasal (Figura 4), evidenciado en el estudio clínico y radiográfico.

Previo a la cirugía, se obtuvo el consentimiento informado del paciente de forma verbal y escrita, aceptando los riesgos y posibles complicaciones del procedimiento.

\section{Presentación de segundo caso}

Paciente masculino 30 años, con historia de ocho horas de haber sufrido un accidente de tránsito. Al historial médico, el paciente se presentó consciente, orientado, con dolor severo bilateral en región mandibular y edema mandibular bilateral con imposibilidad a la apertura bucal. Radiográficamente presentó una fractura de sínfisis y cóndilos mandibulares. Se interconsulta con la especialidad de cirugía maxilofacial por fractura de sínfisis y doble cóndilo mandibular.

Se realizó una intubación submental previa a la reducción abierta de fracturas mandibulares. Al control radiográfico, se observó buen alineamiento de las fracturas (Figura 5).

Para la presentación de los casos clínicos se cuenta con el acta exp. n. ${ }^{\circ}$ 01/2021 de aprobación del Comité de Ética del Hospital Nacional Rosales.

\section{Discusión}

La intubación nasotraqueal está contraindicada en fracturas de la base del cráneo por la incidencia de invasión intracraneal accidental, posibles fugas de líquido cefalorraquídeo, epistaxis, lesión faríngea, necrosis por presión de las fosas nasales externas, infecciones recurrentes del oído medio, sinusitis, sepsis. Teniendo en cuenta que la mayor parte de traumatismos maxilofaciales son a nivel del tercio medio facial, es importante definir un manejo favorable de la vía aérea ${ }^{2,3,7}$.
Una alternativa frecuente es la traqueostomía, que requiere una disección quirúrgica mayor y manipulación de estructuras anatómicas importantes, así como un mayor tiempo quirúrgico. Las complicaciones más documentadas son hemorragias por lesión de la glándula tiroides y sus vasos adyacentes, enfisema quirúrgico, neumotórax, daño recurrente de los nervios laríngeos, paro cardíaco causado por estimulación del nervio vago, infecciones de heridas, edema pulmonar agudo, estenosis traqueal, fístula traqueoesofágica y cicatrices hipertrófi$\operatorname{cas}^{3,4,6,7,8}$.

La intubación submentoniana fue primero descrita como una técnica alternativa para los casos complejos de trauma facial que permitirá mantener las relaciones oclusales mediante la fijación intermaxilar y en los casos en los que se involucra la pirámide nasal o las fracturas que se extienden hacia la base del cráneo. Se ha descrito también el uso de esta técnica en procedimientos estéticos, como cirugía ortognática, combinada con rinoplastia en un solo tiempo quirúrgico; así también, en pacientes con malformaciones craneofaciales, con hipoplasia de tercio medio facial severa, en quienes, por las características de su malformación, resulta una intubación difícil para realizar el procedimiento quirúrgico ${ }^{1,5}$.

Se ha descrito también la utilidad de la misma para efectuar este tipo de procedimientos de reconstrucción facial y de base de cráneo en pacientes pediátricos ${ }^{9}$. El uso de la técnica se ha diversificado y ha iniciado campos de patologías de cabeza y cuello para grandes resecciones de tumores, apoyándose de una intubación retrógrada asistida con videolaringoscopía ${ }^{10}$.

Algunas contraindicaciones para optar por la técnica son: rechazo del paciente al procedimiento, trauma laringotraqueal, infección del sitio quirúrgico, necesidad de ventilación mecánica prolongada, pacientes politraumatizados con daño neurológico, pacientes con daño torácico que requieren múltiples cirugías. Para estos procedimientos la traqueostomía será la alternativa de elección ${ }^{1,23,7,8,11}$.

A pesar de realizarse la intubación submental con un tiempo de operación promedio de principio a fin de 9.9 minutos, exige habilidad y destreza quirúrgica ${ }^{12,13,14}$.

Por esto, la literatura ha reportado complicaciones relacionadas con una mala técnica ejecutada durante el procedimiento quirúrgico, como: una lesión de la glándula sublingual, lesión al nervio lingual, extubación accidental, avance accidental del tubo endotraqueal dentro de los bronquios y obstrucción del tubo endotraqueal. Duran- 


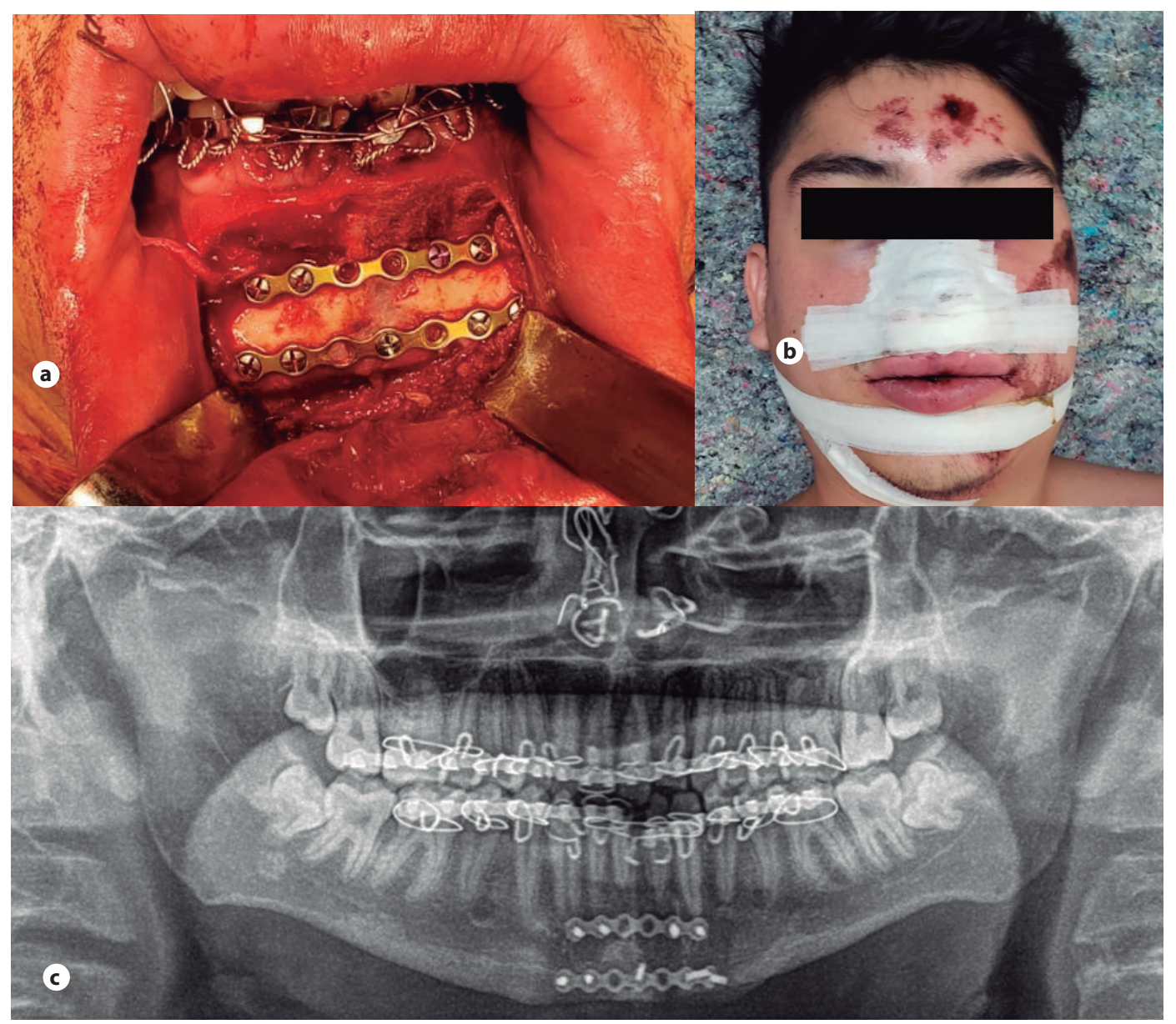

Figura 4a. Reducción de fractura de sínfisis mandibular mediante abordaje vestibular anterior, con la colocación de material de osteosíntesis. 4b. Taponamiento nasal anterior con férula nasal. 4c. Radiografía panorámica control con taponamiento nasal presente, donde se evidencia buena reducción de fractura mandibular.

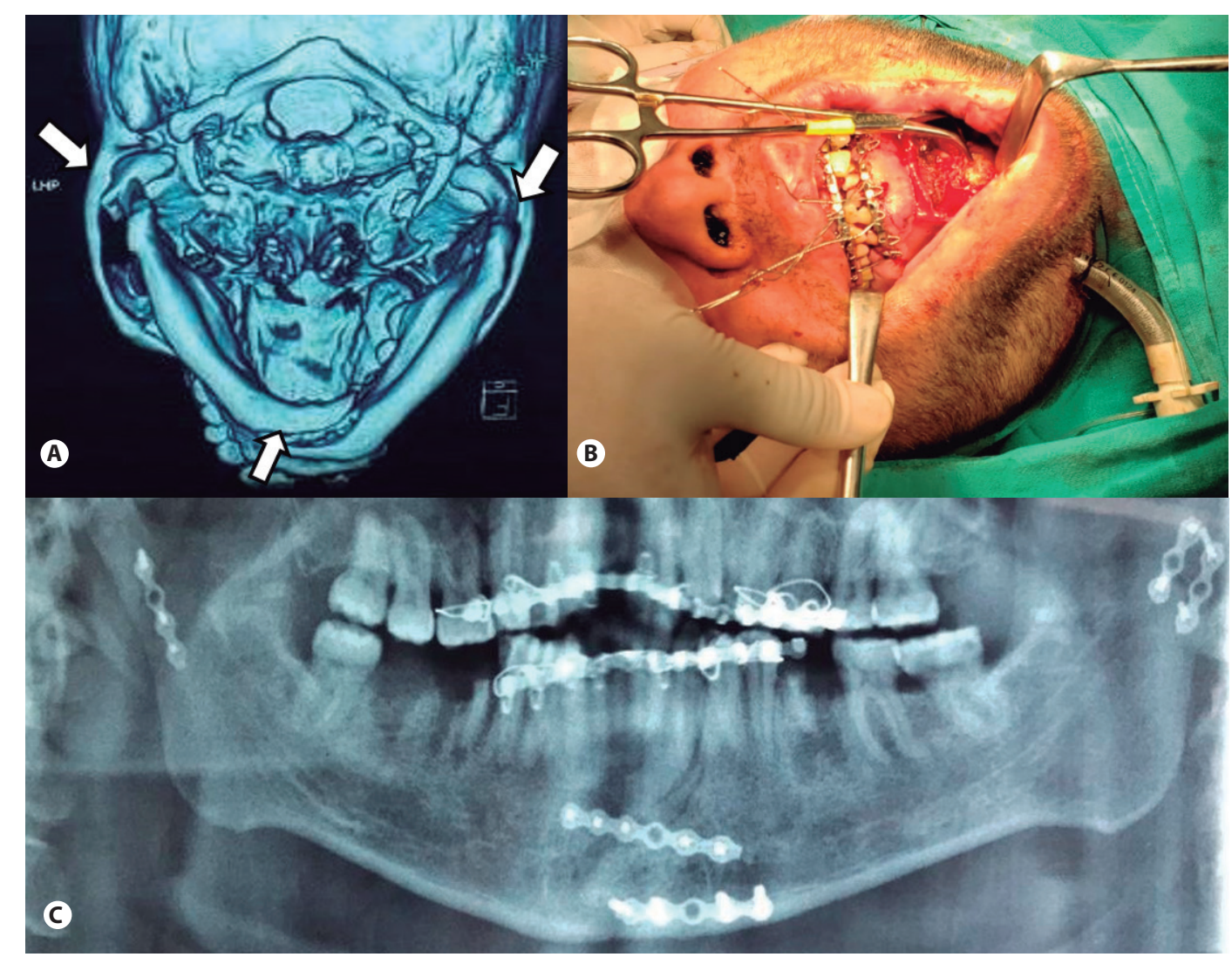

Figura 5a. Fractura de sínfisis y cóndilo mandibular bilateral. 5b. Se realizó la intubación submental, previa a la reducción de fracturas. 5c. Radiografía control posquirúrgica con adecuada reacción de fractura mandibular. 
te el posoperatorio se ha reportado edema, hematoma, fístula mucocutánea, desarrollar mucocele y fístula orocutánea 1,2,3,11,15.

\section{Conclusiones}

La literatura ha demostrado a través de los años que el manejo de la vía aérea mediante intubación submental es una técnica segura y eficaz, pues evita las complicaciones y la morbilidad relacionadas con la traqueostomía. Descrita inicialmente para fracturas complejas faciales, debe considerarse para procedimientos como la cirugía ortognática, las malformaciones severas faciales y para pacientes pediátricos. La ventaja de la técnica descrita en este artículo es que permitió la intubación para una reducción de fractura mandibular y nasal cuando la intubación nasotraqueal no se pudo practicar por defectos anatómicos o patológicos de los pacientes y cuando no fue posible la intubación orotraqueal por la necesidad de establecer las óptimas relaciones maxilomandibulares con bloqueo intermaxilar.

\section{Financiamiento}

El costo de la investigación ha sido asumido por los autores.

\section{Referencias bibliográficas}

1. Navas-Aparicio M del C, Díaz y Faz R. Intubación submentoniana: una alternativa útil para cirugía ortognática en pacientes con malformaciones craneofaciales. Informe de caso. Rev Colomb Anestesiol. 2017;45(S 1):50-4. DOl: 10.1016/j.rca.2016.07.001

2. Licéaga-Escalera CJ, Montoya-Pérez LA, Vélez-Cruz ME, Montoya-Pérez BI. Derivación submental, una alternativa para el manejo de la vía aérea en pacientes con trauma facial. Rev Hosp Jua Mex. 2015;82:169-72. Disponible en: https://www.medigraphic. com/pdfs/juarez/ju-2015/ju153-4e.pdf

3. González-Magaña F, Malagón-Hidalgo HO, García-Cano E, Vilchis-López R, Fentanes-Vera A, Ayala-Ugalde FA. Airway management through submental derivation: a safe and easily reproduced alternative for patients with complex facial trauma. J Korean Assoc Oral Maxillofac Surg. 2018;44(1):12-17. DOI: 10.5125/ jkaoms.2018.44.1.12

4. Bernard AC, Kenady DE. Conventional surgical tracheostomy as the preferred method of airway management. J Oral Maxillofac Surg. 1999;57(3):310-15. DOI: 10.1016/S0278-2391(99)90679-1

5. Hernández Altemir F. The submental route for endotracheal intubation. A new technique. J Maxillofac Surg. 1986;14(1):6465. DOI: 10.1016/S0301-0503(86)80261-2

6. Oshima N, Shiraishi T, Kawauchi T, Oba J, Sato D, Fujiki M, et al. A simple and reliable submental intubation technique for maxillofacial fractures. J Craniofac Surg. 2018;29(7):1952-55. DOI: 10.1097/ SCS.0000000000004628

7. Mishra R, Yadav D, Tripathi S, Kandel L, Baral PP, Shubham S, et al. Submental intubations in panfacial fractures. Clin Cosmet Investig Dent. 2020;12:41-48. DOI: 10.2147/CCIDE. S228326

8. Luce EA, Alvarez SM. Submental intubation. Plast Reconstr Surg Glob Open. 2018;6(9):e1896. DOI: 10.1097/ GOX.0000000000001896

9. Alejandri-Gamboa V, Téllez-Rodríguez PJ, López-Fernández MCR, Sanjuan-Martínez A, Sarmiento L. Submental intubation in pediatric maxillofacial surgery: report of 2 cases. Colomb J Anesthesiol. 2020;48(2):91-95. DOI: 10.1097/ CJ9.0000000000000137

10. Velasco I, Vahdani S. Retrograde Submental Intubation Assisted with Direct Video Laryngoscopy in Maxillofacial Trauma: Technical Note and Case Report. Int J Odontostomat. 2017;11(1):67-70. DOl: 10.4067/S0718-381X2017000100010

11. Lim D, Ma BC, Parumo R, Shanmuhasuntharam $P$. Thirty years of submental intubation: a review. Int J Oral Maxillofac Surg. 2018;47(9):1161-65. DOI: 10.1016/j.ijom.2018.04.015

12. Rodrigues WC, Morais de Melo W, de Almeida RS, Pardo-Kaba SC, Koogi Sonoda C, Hitoshi Shinohara E. Submental intubation in cases of panfacial fractures: a retrospective study. Anesth Prog. 2017;64(3):153-61. DOI: 10.2344/ anpr-64-04-07

13. Jung I, Yoo BH, Ju JY, Choi S, Yon JH, Kim K-M, et al. Novel alternative for submental intubation-A case report. Anesth Pain Med. 2020;15(2):247-50. DOI: 10.17085/ apm.2020.15.2.247

14. Jacob DD, Tuncer FB, Kashan DL, Gurunluoglu R. Clinical Anatomy of Submental Intubation: A Review of the Indications, Technique, and a Modified Approach. Ann Plast Surg. 2020;84(2):232-37 DOI: 10.1097/SAP.0000000000001948

15. Bhola N, Bhutekar U, Jadhav A, Kala A, Deshmukh R. Prasad GSV. Anterior Submandibular Approach for Transmylohyoid Endotracheal Intubation : A Reappraisal with Prospective Study in 206 Cases of Craniomaxillofacial Fractures. Craniomaxillofac Trauma Reconstr. 2017;10(4):255-62. DOI: 10.1055/s-00371607063. 